\title{
AMENDMENTS
}

\section{Author Correction: A brief history of nuclear fusion}

Matteo Barbarino

Correction to: Nature Physics https://doi.org/10.1038/s41567-020-0940-7, published online 11 June 2020.

In the version of this Comment originally published, the second sentence of the section "The stellarator's comeback and the rise of the private sector" referred to the "the 28th FEC"; it should have read "the 27th FEC". All versions of the Comment have been amended.

Published online: 18 June 2020

https://doi.org/10.1038/s41567-020-0966-X

๑ Springer Nature Limited 2020

\section{Author Correction: Polarizing an antiferromagnet by optical engineering of the crystal field}

Ankit S. Disa ID, Michael Fechner, Tobia F. Nova, Biaolong Liu, Michael Först, Dharmalingam Prabhakaran, Paolo G. Radaelli and Andrea Cavalleri (D)

Correction to: Nature Physics https://doi.org/10.1038/s41567-020-0936-3, published online 22 June 2020.

In the version of this Article originally published online, in Fig. 3a, the red text label originally read ' $E \| a$ '; it should have been ' $E \| b$ '. All versions of the Article have been amended.

Published online: 30 June 2020

https://doi.org/10.1038/s41567-020-0977-7

๑) The Author(s), under exclusive licence to Springer Nature Limited 2020

\section{Author Correction: Human information processing in complex networks}

Christopher W. Lynn, Lia Papadopoulos, Ari E. Kahn (D) and Danielle S. Bassett (D)

Correction to: Nature Physics https://doi.org/10.1038/s41567-020-0924-7, published online 15 June 2020.

In the version of this Article originally published online, in the section 'Efficient communication is driven by hierarchically modular structure' the equation for entropy in the second sentence was incorrectly given as $S=\sum_{i} k_{i} \log k_{i}$ and should have been $S=\frac{1}{2 E} \sum_{i} k_{i} \log k_{i}$. All versions of the Article have been amended.

Published online: 7 July 2020

https://doi.org/10.1038/s41567-020-0985-7

(C) The Author(s), under exclusive licence to Springer Nature Limited 2020 